\title{
Long Distance Entanglement of Purification and Reflected Entropy in Conformal Field Theory
}

\author{
Hugo A. Camargo $\odot,{ }^{1,2, *}$ Lucas Hackl, ${ }^{3, \dagger}$ Michal P. Heller ${ }^{1,+}$ Alexander Jahn $\oplus^{2,4, \S}$ and Bennet Windt ${ }^{5, \|}$ \\ ${ }^{1}$ Max-Planck-Institut für Gravitationsphysik, Am Mühlenberg 1, Potsdam-Golm 14476, Germany \\ ${ }^{2}$ Dahlem Center for Complex Quantum Systems, Freie Universität Berlin, Arnimallee 14, Berlin 14195, Germany \\ ${ }^{3}$ School of Mathematics and Statistics \& School of Physics, The University of Melbourne, Parkville, VIC 3010, Australia \\ ${ }^{4}$ Institute for Quantum Information and Matter, California Institute of Technology, Pasadena, California 91125, USA \\ ${ }^{5}$ Blackett Laboratory, Imperial College London, Prince Consort Road, London SW7 2AZ, United Kingdom
}

(Received 17 March 2021; revised 28 June 2021; accepted 23 August 2021; published 29 September 2021)

\begin{abstract}
Quantifying entanglement properties of mixed states in quantum field theory via entanglement of purification and reflected entropy is a new and challenging subject. In this work, we study both quantities for two spherical subregions far away from each other in the vacuum of a conformal field theory in any number of dimensions. Using lattice techniques, we find an elementary proof that the decay of both the entanglement of purification and reflected entropy is enhanced with respect to the mutual information behavior by a logarithm of the distance between the subregions. In the case of the Ising spin chain at criticality and the related free fermion conformal field theory, we compute also the overall coefficients numerically for the both quantities of interest.
\end{abstract}

DOI: 10.1103/PhysRevLett.127.141604

Introduction.-Understanding quantum information properties of quantum field theory (QFT) and, through holography [1-3], also of gravity has been an important contemporary line of research [4-8]. The main object of interest has been the entanglement entropy (EE), which reliably quantifies pure state entanglement between a subregion $A$ and its complement $\bar{A}$. Given a reduced density matrix $\rho_{A}=\operatorname{tr}_{\bar{A}} \rho$ for a total pure state with density matrix $\rho, \mathrm{EE}$ is defined as the von Neumann entropy

$$
S_{A}=S\left(\rho_{A}\right) \equiv-\operatorname{tr}_{A} \rho_{A} \log \rho_{A} .
$$

$\mathrm{EE}$ is an ultraviolet-divergent quantity due to correlations at arbitrarily short distances in QFT and requires a regulator. Efficient computations are possible using Gaussian techniques [9-13] for free QFTs, analytical continuation methods for two-dimensional conformal field theory (CFT) [14-19], or tensor network constructions for both gapped and gapless two-dimensional systems [20,21]. In strongly coupled holographic QFTs, computing EE reduces to a geometric problem of finding minimal surfaces [22-25].

In the present work, we will be concerned with CFTs in arbitrary number of dimensions emerging as a longdistance limit of lattice models regulated by a lattice spacing $\delta$. We will be interested in entanglement for

Published by the American Physical Society under the terms of the Creative Commons Attribution 4.0 International license. Further distribution of this work must maintain attribution to the author(s) and the published article's title, journal citation, and DOI. Funded by SCOAP ${ }^{3}$. subsystems composed of two disjoint regions $A$ and $B$, for which one often considers the mutual information (MI) defined as

$$
I(A: B)=S_{A}+S_{B}-S_{A B} .
$$

A quantity of significant recent interest in such a setup is also the entanglement of purification (EOP) [26], which can be regarded as a generalization of $\mathrm{EE}$ for bipartite mixed states. It requires purifying the reduced density matrix $\rho_{A B}$ to a pure state $|\psi\rangle$ in an enlarged Hilbert space on $\mathcal{H}_{A B} \rightarrow \mathcal{H}_{A A^{\prime} B B^{\prime}}$ such that $\rho_{A B}=\operatorname{tr}_{A^{\prime} B^{\prime}}|\psi\rangle\langle\psi|$ (visualized in Fig. 1). The EOP is then defined as

$$
E_{P}\left(\rho_{A B}\right)=\min _{\psi}\left[S_{A A^{\prime}}\right] \text {. }
$$

EOP is challenging to compute in QFT due to its inherent optimization procedure of finding a purification whose $\mathrm{EE}$ is minimal. Its current understanding in the intersection of quantum information and high-energy physics is based on Gaussian calculations [27-29], CFT techniques with a limited range of applicability [30-32], and on a conjectured realization in holography $[33,34]$. In the latter case, EOP has been conjectured to be dual to the entanglement wedge cross section [35-38]. This led to many novel developments regarding the emergence of the gravitational hologram [39], see, e.g., [40-50].

Another quantity closely related to EOP and also conjectured to be holographically dual to the entanglement wedge cross section is the reflected entropy (RE) [48,5160]. It is defined as EE

$$
S_{R}\left(\rho_{A B}\right)=S_{A A^{\prime}}\left(\left|\sqrt{\rho_{A B}}\right\rangle\right)
$$




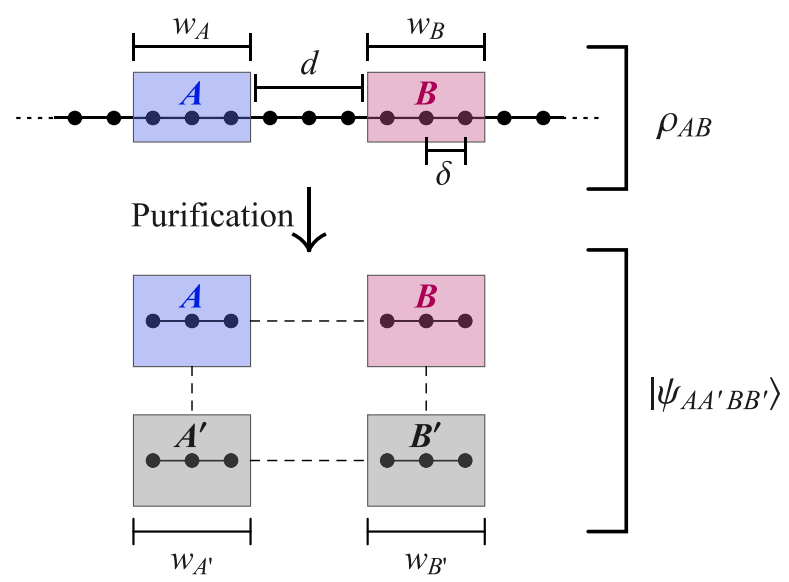

FIG. 1. Illustration of our general setup for CFTs in two spacetime dimensions on a lattice. The mixed state $\rho_{A B}$ on a subsystem of two disjoint regions $A B$ separated by $N_{d} \equiv(d / \delta)$ sites is purified to a state with auxiliary factors $A^{\prime}$ and $B^{\prime}$, taken to be of the same size $N_{A} \equiv w_{A} / \delta$ and $N_{B} \equiv w_{B} / \delta$ as $A$ and $B$, respectively, where $\delta$ is the lattice spacing. Here we mostly consider $N_{A}=N_{B}$.

of the unique purification $\left|\sqrt{\rho_{A B}}\right\rangle:=\sum_{i} \sqrt{e_{i}}\left|e_{i}\right\rangle\left|e_{i}\right\rangle \in$ $\mathcal{H}_{A} \otimes \mathcal{H}_{B} \otimes \mathcal{H}_{A^{\prime}} \otimes \mathcal{H}_{B^{\prime}}$ of $\rho_{A B}$, where $\rho_{A B}\left|e_{i}\right\rangle=e_{i}\left|e_{i}\right\rangle$, $\mathcal{H}_{A^{\prime}}=\mathcal{H}_{A}$, and $\mathcal{H}_{B}=\mathcal{H}_{B^{\prime}}$. Simply put, $\left|\sqrt{\rho_{A B}}\right\rangle$ is the unique purification symmetric under $A \leftrightarrow A^{\prime}$ and $B \leftrightarrow B^{\prime}$ exchange. Clearly, $\left|\sqrt{\rho_{A B}}\right\rangle$ is one of the valid purifications $|\psi\rangle$ we minimize over in (3), which implies $E_{P} \leq S_{R}$. RE is much easier to compute compared to EOP, as it does not require an optimization over all possible purifications.

The aim of this letter is to elucidate a particularly simple setting in which EOP and RE behave universally across CFTs, without relying on Gaussianity or Weyl rescalings. We achieve this by using spin chains and more general lattice models and focusing on universal inequalities satisfied by EE. We corroborate our studies using analytics and numerics in the Ising and free fermion CFTs [61], which allows us to extract prefactors in the asymptotic scaling of EOP and RE.

Setup.-In our analysis, we will be concerned with CFTs on a lattice. Our general statements will be made in any number of dimensions, whereas our numerics will focus on CFTs in two spacetime dimensions.

The setting of interest will contain two spherical subregions of diameter $w$ separated by a distance $d$. Figure 1 illustrates it for CFTs in two spacetime dimensions in which case the subregions become intervals. At large distances $(d / w) \gg 1$, the decay of MI (2) in CFTs reads

$$
I(A: B)=\mathcal{N} \frac{\Gamma\left(\frac{3}{2}\right) \Gamma(2 \Delta+1)}{2^{4 \Delta+1} \Gamma\left(2 \Delta+\frac{3}{2}\right)} \times \epsilon_{\Delta}^{2}+\ldots,
$$

where

$$
\epsilon_{\Delta} \equiv\left(\frac{w}{d}\right)^{2 \Delta}
$$

and $\Delta$ corresponds of the scaling dimension of the lowest nontrivial operator(s) in the theory, $\mathcal{N}$ denotes the possible degeneracy of such operators, and the ellipsis denotes faster decaying terms $[18,19,62,63]$. The formula (5) assumes a gap in the spectrum of scaling dimensions and the lowest lying operator(s) being scalar(s). We will carry over this assumption in our studies of EOP and RE.

Our aim is to find and prove an analog of the scaling in (5) for EOP and RE. In the latter case, recent numerical studies in free CFTs in $[56,57]$ led to the following fit:

$$
S_{R}=\alpha \epsilon_{\Delta}^{2} \log \left(\epsilon_{\Delta}^{-2}\right)+\ldots \text { for } \epsilon_{\Delta} \ll 1,
$$

where $\alpha$ is a positive model-dependent constant.

In this Letter, we use quantum-many body techniques in conjunction with elementary EE inequalities to prove that the asymptotic form (7) holds both for EOP and RE in a general CFT amenable to a lattice realization.

Elementary proof of the large-distance behavior.-To set up the general argument valid both for EOP and RE, we only need to assume that the density operator $\rho_{A B}$ of two subsystems $A$ and $B$ far away from each other takes the form

$$
\rho_{A B}\left(\epsilon_{\Delta}\right)=\rho_{A}^{(0)} \otimes \rho_{B}^{(0)}+\epsilon_{\Delta} \rho_{A B}^{(1)}+\frac{1}{2} \epsilon_{\Delta}^{2} \rho_{A B}^{(2)}+, \ldots,
$$

where the ellipsis denotes higher, not necessarily integer powers of $\epsilon_{\Delta}$ and we do not make any assumptions about subsystem sizes. The $\epsilon_{\Delta}$ term in (8) is needed to reproduce the power-law scaling of correlation functions involving insertions of the lowest lying scaling operator in both $A$ and $B$. As we will show, the $\rho_{A B}^{(2)}$ contribution turns out to not contribute to the leading order decay of EOP and RE.

In the following, we will regard (8) as originating from a perturbative purification

$$
|\psi\rangle=\left|\psi^{(0)}\right\rangle+\epsilon_{\Delta}\left|\psi^{(1)}\right\rangle+\frac{1}{2} \epsilon_{\Delta}^{2}\left|\psi^{(2)}\right\rangle+, \ldots,
$$

where the product nature of the density matrix (8) for an infinite separation leads to

$$
\left|\psi^{(0)}\right\rangle=\left|\psi_{A A^{\prime}}^{(0)}\right\rangle \otimes\left|\psi_{B B^{\prime}}^{(0)}\right\rangle .
$$

Note that, in our conventions, $|\psi\rangle$ and, therefore, also $\left|\psi^{(0)}\right\rangle$ are normalized, which also leads to constraint for $\left|\psi^{(j \geq 1)}\right\rangle$. The long distance behavior of EOP and RE is determined by the small- $\epsilon_{\Delta}$ expansion of the eigenvalues $\mu_{j}$ of

$$
\rho_{A A^{\prime}} \equiv \operatorname{tr}_{B B^{\prime}}|\psi\rangle\langle\psi|
$$

via the definition of $\mathrm{EE}(1): S_{A A^{\prime}}(|\psi\rangle)=-\sum_{j \geq 0} \mu_{j} \log \mu_{j}$.

The fact that $\rho_{A B}$ is a product state for $\epsilon_{\Delta}=0$ implies that $\rho_{A A^{\prime}}\left(\epsilon_{\Delta}=0\right)$ is itself pure, see (10), and thus has eigenvalues $\mu_{0}=1$ and $\mu_{j>0}=0$. This result gets modified at large but finite distances.

The linear correction to $\mu_{j}$ vanishes, since we expect $\mu_{j}$ to originate from a well-defined density matrix regardless of the sign of $\epsilon_{\Delta}$ when viewed as a formal parameter. As a 
result, the possible leading behavior of eigenvalues of $\rho_{A A^{\prime}}$ is given by

$$
\mu_{0} \sim 1-\alpha_{\mathrm{tot}} \epsilon_{\Delta}^{2} \quad \text { and } \quad \mu_{j>0} \sim \alpha_{j} \epsilon_{\Delta}^{2} \quad \text { as } \epsilon_{\Delta} \rightarrow 0,
$$

where

$$
\alpha_{\mathrm{tot}} \equiv \sum_{j>0} \alpha_{j}
$$

Note that $\alpha_{j>0} \geq 0$ and if all of them vanished, the behavior encapsulated by (12) would simply involve a higher-thantwo power of $\epsilon_{\Delta}$.

Let us consider now the asymptotics of EOP and RE resulting from (12). As we explained in the introduction, these quantities are given by $S_{A A^{\prime}}$ subject to additional conditions on purifications. For any purification leading to (12), $S_{A A^{\prime}}$ behaves as

$$
S_{A A^{\prime}}=\alpha_{\mathrm{tot}} \epsilon_{\Delta}^{2} \log \epsilon_{\Delta}^{-2}+\beta \epsilon_{\Delta}^{2}+, \ldots,
$$

where

$$
\beta \equiv\left(\sum_{j>0} \alpha_{j}\left(1-\log \alpha_{j}\right)\right)
$$

and one sees as the leading order behavior the structure (7) identified in fits to free CFTs RE numerics in $[56,57]$ and the ellipsis denotes higher order terms in $\epsilon_{\Delta}$.

Regardless of purification and long-distance limit, $S_{A A^{\prime}}$ is bounded from below [64]

$$
S_{A A^{\prime}}(|\psi\rangle) \geq \frac{1}{2} I_{A B}\left(\rho_{A B}\right),
$$

as was shown in Eq. (6) of [65]. Given (5), in order for (16) to be satisfied at large distances $S_{A A^{\prime}}$ cannot scale with a higher power than $\epsilon_{\Delta}^{2}$. Since the eigenvalue analysis predicts this as the strongest possible power-law factor in the long-distance behavior of $S_{A A^{\prime}}, \alpha_{\text {tot }}$ must be bigger than 0 , and the behavior predicted by (14) is necessarily the behavior of both EOP and RE in any CFT with a gap in the operator spectrum and amenable to a lattice description.

As a corollary of this proof, from the definition of $\alpha_{\text {tot }}$ in (13) we necessarily obtain that at least one of $\alpha_{j>0}>0$ and, as a result, the first subleading term encapsulated in (14) is also generically there. This is consistent with the findings of [56,57], which also identified such a contribution in RE for free CFTs on a lattice.

Finally, let us emphasize that our proof of the longdistance behavior of EOP and RE did not rely on dimensionality of a CFT in question.

Properties of the overall coefficient.-Our proof predicts only that the overall prefactor $\alpha_{\text {tot }}$ is positive. It is possible, however, to extract more information about what ingredients affect the exact value of $\alpha_{\text {tot }}$ using a rather general argument. To this end, notice that perhaps the easiest way to compute $\alpha_{\text {tot }}$ is to extract it from

$$
\operatorname{Tr}\left(\rho_{A A^{\prime}}^{2}\right)=1-2 \alpha_{\mathrm{tot}} \epsilon_{\Delta}^{2}+\ldots,
$$

where we suppressed higher order terms in $\epsilon_{\Delta}$.

Starting with (9) and defining

$$
\left|\psi_{A A^{\prime}}^{(i)}\right\rangle=\left(\mathbb{1} \otimes\left\langle\psi_{B B^{\prime}}^{(0)}\right|\right)\left|\psi^{(i)}\right\rangle,
$$

we can write the reduction $\rho_{A A^{\prime}}$ as

$$
\begin{aligned}
\rho_{A A^{\prime}}= & \left|\psi_{A A^{\prime}}^{(0)}\right\rangle\left\langle\psi_{A A^{\prime}}^{(0)}\right|+\epsilon_{\Delta}\left(\left|\psi_{A A^{\prime}}^{(0)}\right\rangle\left\langle\psi_{A A^{\prime}}^{(1)}|+| \psi_{A A^{\prime}}^{(1)}\right\rangle\left\langle\psi_{A A^{\prime}}^{(0)}\right|\right) \\
& +\frac{1}{2} \epsilon_{\Delta}^{2}\left(2 \operatorname{Tr}_{B B^{\prime}}\left|\psi^{(1)}\right\rangle\left\langle\psi^{(1)}|+| \psi_{A A^{\prime}}^{(2)}\right\rangle\left\langle\psi_{A A^{\prime}}^{(0)}\right|\right. \\
& \left.+\left|\psi_{A A^{\prime}}^{(0)}\right\rangle\left\langle\psi_{A A^{\prime}}^{(2)}\right|\right),
\end{aligned}
$$

which allows us to compute $\operatorname{Tr}\left(\rho_{A A^{\prime}}^{2}\right)$ explicitly. Upon using the normalization condition $\langle\psi \mid \psi\rangle=1$ in the form of the following constraints

$$
\begin{gathered}
\left\langle\psi^{(0)} \mid \psi^{(1)}\right\rangle+\left\langle\psi^{(1)} \mid \psi^{(0)}\right\rangle=0, \\
\left\langle\psi^{(0)} \mid \psi^{(2)}\right\rangle+\left\langle\psi^{(2)} \mid \psi^{(0)}\right\rangle+2\left\langle\psi^{(1)} \mid \psi^{(1)}\right\rangle=0,
\end{gathered}
$$

we obtain

$\alpha_{\mathrm{tot}}=\|\left|\psi^{(1)}\right\rangle\left\|^{2}+\left|\left\langle\psi^{(0)} \mid \psi^{(1)}\right\rangle\right|^{2}-\right\|\left|\psi_{A A^{\prime}}^{(1)}\right\rangle\left\|^{2}-\right\|\left|\psi_{B B^{\prime}}^{(1)}\right\rangle \|^{2}$,

where, in analogy with (18), $\left|\psi_{B B^{\prime}}^{(1)}\right\rangle \equiv\left(\left\langle\psi_{A A^{\prime}}^{(0)}\right| \otimes \mathbb{1}\right)\left|\psi^{(1)}\right\rangle$.

Quite remarkably and as advertised below (8), the correction quadratic in $\epsilon_{\Delta}$ to the density matrix $\rho_{A B}$ does not contribute to the leading order coefficient in the scaling of both EOP and RE. This looks like a potentially useful insight for any attempt to fix $\alpha_{\text {tot }}$ for EOP and RE in terms of CFT data in an analogous manner to (5) for MI. Furthermore, obtaining the leading behavior of the EOP amounts simply to minimizing a quadratic polynomial obtained from components of $\left|\psi^{(1)}\right\rangle$ subject to the constraint (20) and the condition [with $\rho_{A B}^{(1)}$ from (8)]

$$
\rho_{A B}^{(1)}=\operatorname{Tr}_{B^{\prime} A^{\prime}}\left(\left|\psi^{(1)}\right\rangle\left\langle\psi^{(0)}|+| \psi^{(0)}\right\rangle\left\langle\psi^{(1)}\right|\right),
$$

which generally leads to affine-linear constraints on $\left|\psi^{(1)}\right\rangle$. The fact that the minimum exists follows from the argument presented in the previous section. While $\rho_{A B}^{(2)}$ does not affect the leading large-distance behavior encapsulated by $\alpha_{\text {tot }}$, individual $\alpha_{j>0}$ do depend on it and, via (14), so does the coefficient in front of the subleading term quadratic in $\epsilon_{\Delta}$.

Analysis in the critical Ising chain and free fermion CFT.-So far, we have been completely general in our studies and in the following we will specialize to two closely related lattice models describing CFTs in two spacetime dimensions. This will allow us to obtain numerical values of the leading and first subleading coefficients in the behavior of EOP and RE captured by (14) and, for RE, compare with earlier studies in [56]. 
The Ising model realization of the $c=\frac{1}{2} \mathrm{CFT}$ on an infinite spatial line can be described by the critical lattice Hamiltonian

$$
\hat{H} \sim-\sum_{i=-\infty}^{\infty}\left(2 \hat{S}_{i}^{x} \hat{S}_{i+1}^{x}+\hat{S}_{i}^{z}\right),
$$

more general forms of which we discuss in the Supplemental Material [66]. The $\hat{S}_{i}^{x, z}$ are spin operators defined by the Pauli matrices $\hat{S}_{i}^{x, z}=\frac{1}{2} \sigma_{i}^{x, z}$. In the Ising CFT there is a nondegenerate (i.e., $\mathcal{N}=1$ ) lightest operator of scaling dimension $\Delta=1 / 8$, often denoted as the spin field $\sigma$ and corresponding to a $\hat{S}_{i}^{x}$ lattice operator.

The critical Ising model can be mapped to a free fermion theory, which is going to be another model in which we

Free fermions
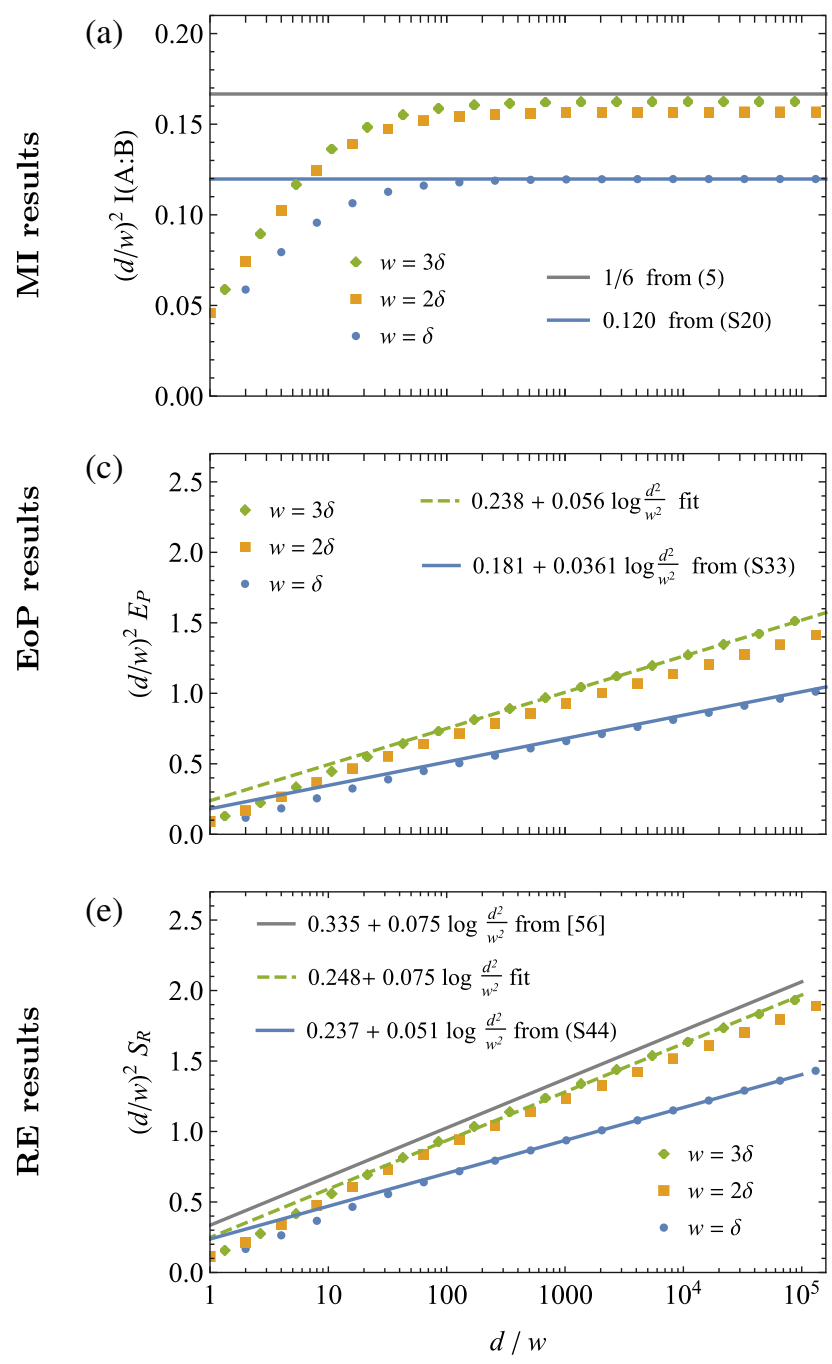

obtain numerical coefficients in EOP and RE. This formulation leads to two different notions of reduced density matrices for disjoint intervals, see, for example, [29,70-73], and therefore provides in itself an independent example. For free fermion CFT there are two $(\mathcal{N}=2)$ lowest lying operators with $\Delta=1 / 2$ and being simply the fermionic field operators.

Critical lattice models will describe CFT predictions for large enough sizes of subsystems at fixed $w / d$. Since we are dealing with purifications, which can lead to challenges when the relevant Hilbert space dimension becomes big, the key question is how big subsystems need to be to reproduce the continuum physics of interest. One hint comes from MI, for which we see that the continuum value of the prefactor in (5) is well attained at large distances already for $w=2 \delta$ and

Ising spins

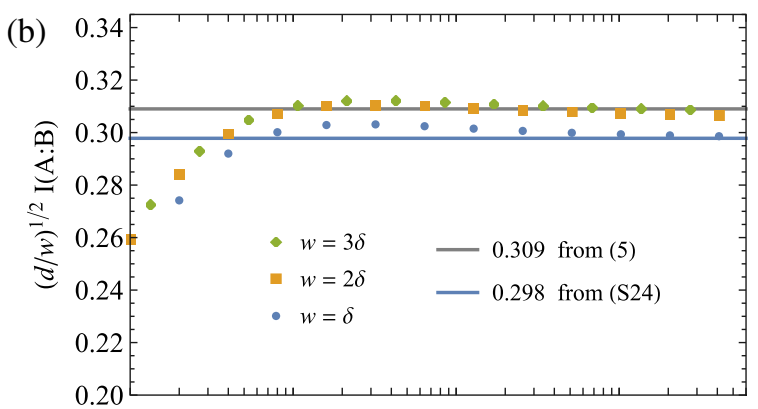

(d)
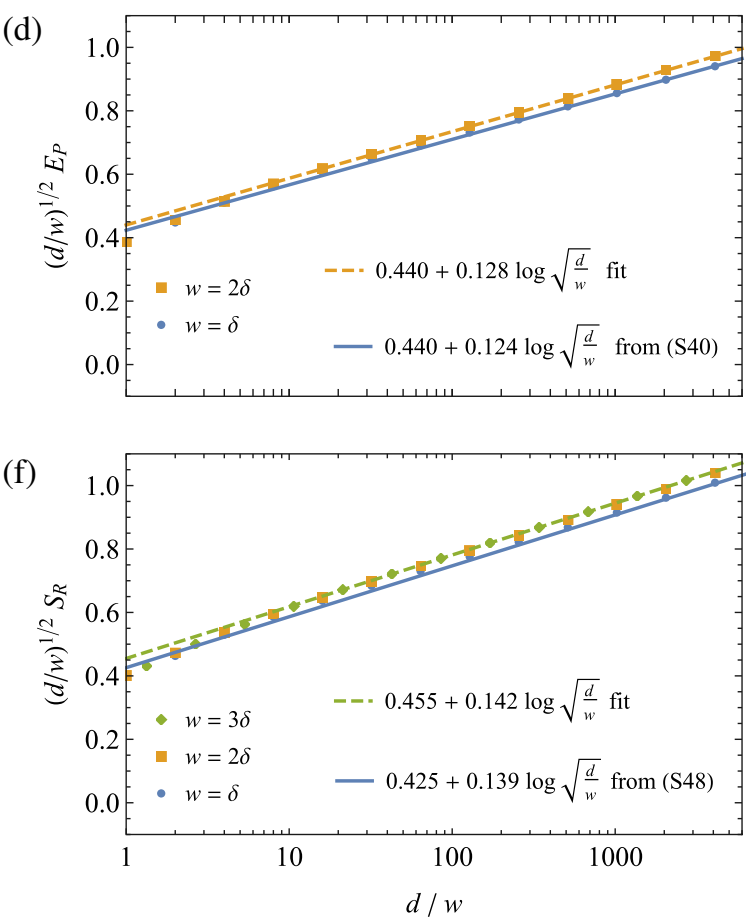

FIG. 2. Numerical data for MI, EOP, and RE for fermions and spins, rescaled by the power-law contribution $\epsilon_{\Delta}^{2}=(w / d)^{4 \Delta}$ of the respective dominant term with $\Delta=1 / 2$ for free fermions and $\Delta=1 / 8$ for Ising spins. The analytical predictions for $w=\delta$ are derived in the Supplemental Material [66] (see also Table I). Analytical comparisons are drawn as solid lines and fits of the numerical EOP and RE data at the largest available $w$ as dashed ones. The top solid (gray) line in (e) displayed above the numerical data corresponds to the result reported in [56] for the continuum $(\delta \rightarrow 0)$. 
TABLE I. Summary of numerical and analytical results for the leading coefficient $\alpha_{\mathrm{tot}}$ and the offset $\beta$ obtained for MI, EOP and RE with asymptotics (14) both for Ising spins and for latticized fermions on a line. We refer to the respective equation in the Supplemental Material [66]. Numbers without analytical expression are based on a numerical fit.

\begin{tabular}{|c|c|c|c|c|c|c|}
\hline & \multicolumn{3}{|c|}{ Free Fermions (Gaussian) } & \multicolumn{3}{|c|}{ Ising Spins (non-Gaussian) } \\
\hline & Coefficient $\alpha_{\text {tot }}$ & Offset $\beta$ & Equation & Coefficient $\alpha_{\text {tot }}$ & Offset $\beta$ & Equation \\
\hline MI & 0 & $\begin{array}{c}\{\log [\pi+2 / \pi-2] / 4 \pi\} \\
\approx 0.120\end{array}$ & $(\mathrm{~S} 20)$ & 0 & $\begin{array}{c}C^{2}\left\{\left[4 \pi^{2} /\left(\pi^{2}-4\right)\right]\right. \\
+(\pi / 2) \log \left[\left(4+4 \pi+\pi^{2}\right) /\right. \\
\left.\left.\left(4-4 \pi+\pi^{2}\right)\right]\right\} \approx 0.298\end{array}$ & $(\mathrm{~S} 24)$ \\
\hline EOP & $\begin{array}{c}{\left[1 /\left(8+2 \pi^{2}\right)\right]} \\
\approx 0.036\end{array}$ & $\begin{array}{c}\left\{\left[\log 2 e\left(8+2 \pi^{2}\right)\right] /\left(8+2 \pi^{2}\right)\right\} \\
\approx 0.181\end{array}$ & (S33) & $\begin{array}{c}{\left[4 C^{2} \pi^{4} /\left(\pi^{4}-16\right)\right.} \\
\approx 0.124\end{array}$ & 0.440 & $(\mathrm{~S} 40)$ \\
\hline RE & $\left(1 / 2 \pi^{2}\right) \approx 0.051$ & $\begin{array}{c}\left\{\left[1+\log \left(4 \pi^{2}\right)\right] / 2 \pi^{2}\right\} \\
\approx 0.237\end{array}$ & $(\mathrm{~S} 44)$ & $\begin{array}{c}\left\{\left[4 C^{2}\left(\pi^{2}-2\right)\right] /\left(\pi^{2}-4\right)\right\} \\
\approx 0.139\end{array}$ & 0.425 & $(\mathrm{~S} 48)$ \\
\hline
\end{tabular}

$3 \delta$ with the smallest subsystems of $w=\delta$ giving already reasonable predictions, see Figs. 2(a) and 2(b).

Given this encouraging result, we were in fact able to analytically compute the coefficients of the leading order MI, EOP, and RE in the critical Ising model and for free fermions when $w=\delta$. The results are summarized in Table I together with offsets and derivations can be found in the Supplemental Material [66].

Figures 2(c)-2(f) show fits of our proven asymptotic formula (14) to fully numerical results, i.e., based on the full density matrix for disjoint intervals of both CFTs, which we consider. We see strong indications of convergence to continuum values. In particular, for the critical Ising model EOP, the behavior of $\alpha_{\text {tot }}$ for $w=\delta$ corroborates our analytical prediction in Table I. Looking at the results for the largest attainable values of $w$, we see that the leading falloff coefficient changes from the analytic prediction at $w=\delta$ by only $2.6 \%$ and the subleading falloff coefficient by only $3.9 \%$. Generating data for $w=3 \delta$ and above is numerically challenging as this requires computing very large matrices, slowing down calculations (see the Supplemental Material [66]).

For the EOP and RE of the Ising CFT, our results provide to the best of our knowledge new predictions; whereas, for $\mathrm{RE}$ for the massless free fermions, we find and display very good agreement with earlier studies in [56], where the continuum $(\delta \rightarrow 0)$ is studied. The remaining difference is due to the fact that $w=3 \delta$ does not fully capture this continuum limit.

An interesting question regarding EOP concerns the dimensions of the enlarged Hilbert spaces. In our setup, when purifying the state of a system with $N_{A}+N_{B}$ degrees of freedom by adding $N_{A^{\prime}}+N_{B^{\prime}}$ additional ones (see also Fig. 1), there is a priori no constraint on $N_{A^{\prime}}, N_{B^{\prime}}$ other than the basic requirement following from the definition of the Schmidt decomposition that $N_{A^{\prime}}+N_{B^{\prime}} \geq N_{A}+N_{B}$. However, we show in the Supplemental Material [66] that the choice of minimal purifications used so far yields the true minimum of EE as long as we choose $N_{A^{\prime}}=N_{A}$ and $N_{B^{\prime}}=N_{B}$. This was already shown in [74] based on ideas of [75] for the EOP for Gaussian states (with Gaussian purifications).

Outlook.-In this Letter, we studied large-distance behavior of EOP and RE in a generic CFT with a gap in the operator spectrum for two spherical subsystems of diameter $w$ in the large-distance $d$ limit. Using (8) in conjunction with elementary properties of $\mathrm{EE}$ we were able to prove that the large order behavior of both EOP and RE is governed by (14). In comparison to the classic result (5) encapsulating large-distance behavior of MI, EOP, and RE get both enhanced by a logarithm of a separation. Subsequently, we explicitly calculated the large-distance behavior for both EOP and RE in one spatial dimension for the critical Ising model and massless fermions. This allowed us to establish the value of coefficients appearing in (14), see Table I and Fig. 2.

Our work opens a genuinely new avenue for studying EOP and RE in QFTs without restriction to free models. Perhaps the most interesting question concerns the dependence of the coefficients in the large order behavior of EOP and RE on CFT data, akin to (5) for MI. An intermediate step could be to supplement our numerical code with largedistance reduced density matrices obtained with tensor networks for more complicated models, in particular determining model-dependent coefficients akin to (5). Optimizing over purifications outside the Gaussian realm inevitably leads to vast parameter spaces that quickly exhaust desktop-scale computational resources. However, the entanglement between reasonably sized subsystems both mixed and purified is not large and it should be possible to represent purifications as manageable tensor networks, perhaps building on earlier works $[34,76]$.

We would like to thank J. Eisert and T. Takayanagi for collaborations on related subjects and M.C. Bañuls, T. Faulkner, J. Knaute, C. Pattison, D. Radicevic, L. Shaposhnik, S. Singh, V. Svensson, B. Swingle, and L. Tagliacozzo for useful discussions and comments on the draft. Our special thanks go to P. Bueno who in response to the first version of the manuscript pointed out to us that the 
behavior encapsulated by (7) was also seen in the reflected entropy in free fermion and free boson QFTs [56,57]. The Gravity, Quantum Fields and Information group at the Max Planck Institute for Gravitational Physics (Albert Einstein Institute) is supported by the Alexander von Humboldt Foundation and the Federal Ministry for Education and Research through the Sofja Kovalevskaja Award. A. J. is supported by the FQXi. H. C. is partially supported by the Konrad-Adenauer-Stiftung through their Sponsorship Program for Foreign Students and by the International Max Planck Research School for Mathematical and Physical Aspects of Gravitation, Cosmology and Quantum Field Theory.

*hugo.camargo@aei.mpg.de

†lucas.hackl@unimelb.edu.au

*michal.p.heller@aei.mpg.de; On leave of absence from: National Centre for Nuclear Research, Pasteura 7, 02-093 Warsaw, Poland

$\S$ a.jahn@fu-berlin.de

bennet.windt17@imperial.ac.uk

[1] J. M. Maldacena, The large N limit of superconformal field theories and supergravity, Int. J. Theor. Phys. 38, 1113 (1999); Adv. Theor. Math. Phys. 2, 231 (1998).

[2] S. Gubser, I. R. Klebanov, and A. M. Polyakov, Gauge theory correlators from noncritical string theory, Phys. Lett. B 428, 105 (1998).

[3] E. Witten, Anti-de sitter space and holography, Adv. Theor. Math. Phys. 2, 253 (1998).

[4] H. Casini and M. Huerta, Entanglement entropy in free quantum field theory, J. Phys. A 42, 504007 (2009).

[5] D. Harlow, Jerusalem lectures on black holes and quantum information, Rev. Mod. Phys. 88, 015002 (2016).

[6] M. Rangamani and T. Takayanagi, Holographic Entanglement Entropy, (Springer, New York, 2017), Vol. 931.

[7] L. Susskind, Three lectures on complexity and black holes, arXiv:1810.11563.

[8] M. Headrick, Lectures on entanglement entropy in field theory and holography, arXiv:1907.08126.

[9] R.D. Sorkin, On the entropy of the vacuum outside a horizon, in Proceedings of the Tenth International Conference on General Relativity and Gravitation (held in Padova, 4-9 July, 1983), Contributed Papers (Springer, Dordrecht, 1983), Vol. 2 pp. 734-736.

[10] I. Peschel, Calculation of reduced density matrices from correlation functions, J. Phys. A 36, L205 (2003).

[11] C. Weedbrook, S. Pirandola, R. García-Patrón, N. J. Cerf, T. C. Ralph, J. H. Shapiro, and S. Lloyd, Gaussian quantum information, Rev. Mod. Phys. 84, 621 (2012).

[12] E. Bianchi, L. Hackl, and N. Yokomizo, Entanglement entropy of squeezed vacua on a lattice, Phys. Rev. D 92, 085045 (2015).

[13] L. Hackl and E. Bianchi, Bosonic and fermionic gaussian states from Kähler structures, arXiv:2010.15518.

[14] C. Holzhey, F. Larsen, and F. Wilczek, Geometric and renormalized entropy in conformal field theory, Nucl. Phys. B424, 443 (1994).
[15] P. Calabrese and J. L. Cardy, Entanglement entropy and quantum field theory, J. Stat. Mech. (2004) P06002.

[16] P. Calabrese and J. Cardy, Entanglement entropy and conformal field theory, J. Phys. A 42, 504005 (2009).

[17] P. Calabrese, J. Cardy, and E. Tonni, Entanglement entropy of two disjoint intervals in conformal field theory, J. Stat. Mech. (2009) P11001.

[18] J. Cardy, Some results on the mutual information of disjoint regions in higher dimensions, J. Phys. A 46, 285402 (2013).

[19] T. Ugajin, Mutual information of excited states and relative entropy of two disjoint subsystems in CFT, J. High Energy Phys. 10 (2017) 184.

[20] M. B. Hastings, An area law for one-dimensional quantum systems, J. Stat. Mech. (2007) P08024.

[21] G. Vidal, Class of Quantum Many-Body States That Can Be Efficiently Simulated, Phys. Rev. Lett. 101, 110501 (2008).

[22] S. Ryu and T. Takayanagi, Holographic Derivation of Entanglement Entropy from AdS/CFT, Phys. Rev. Lett. 96, 181602 (2006).

[23] V.E. Hubeny, M. Rangamani, and T. Takayanagi, A covariant holographic entanglement entropy proposal, J. High Energy Phys. 07 (2007) 062.

[24] A. Lewkowycz and J. Maldacena, Generalized gravitational entropy, J. High Energy Phys. 08 (2013) 090.

[25] X. Dong, A. Lewkowycz, and M. Rangamani, Deriving covariant holographic entanglement, J. High Energy Phys. 11 (2016) 028.

[26] B. M. Terhal, M. Horodecki, D. W. Leung, and D. P. DiVincenzo, The entanglement of purification, J. Math. Phys. (N.Y.) 43, 4286 (2002).

[27] A. Bhattacharyya, T. Takayanagi, and K. Umemoto, Entanglement of purification in free scalar field theories, J. High Energy Phys. 04 (2018) 132.

[28] A. Bhattacharyya, A. Jahn, T. Takayanagi, and K. Umemoto, Entanglement of Purification in Many Body Systems and Symmetry Breaking, Phys. Rev. Lett. 122, 201601 (2019).

[29] H. A. Camargo, L. Hackl, M. P. Heller, A. Jahn, T. Takayanagi, and B. Windt, Entanglement and complexity of purification in $(1+1)$-dimensional free conformal field theories, Phys. Rev. Research 3, 013248 (2021).

[30] H. Hirai, K. Tamaoka, and T. Yokoya, Towards entanglement of purification for conformal field theories, Prog. Theor. Exp. Phys. 2018, 063 B03 (2018).

[31] P. Caputa, M. Miyaji, T. Takayanagi, and K. Umemoto, Holographic Entanglement of Purification from Conformal Field Theories, Phys. Rev. Lett. 122, 111601 (2019).

[32] W.-Z. Guo, Entanglement of purification and disentanglement in CFTs, J. High Energy Phys. 09 (2019) 080.

[33] T. Takayanagi and K. Umemoto, Entanglement of purification through holographic duality, Nat. Phys. 14, 573 (2018).

[34] P. Nguyen, T. Devakul, M. G. Halbasch, M. P. Zaletel, and B. Swingle, Entanglement of purification: From spin chains to holography, J. High Energy Phys. 01 (2018) 098.

[35] B. Czech, J. L. Karczmarek, F. Nogueira, and M. Van Raamsdonk, The gravity dual of a density matrix, Classical Quantum Gravity 29, 155009 (2012). 
[36] A. C. Wall, Maximin surfaces, and the strong subadditivity of the covariant holographic entanglement entropy, Classical Quantum Gravity 31, 225007 (2014).

[37] M. Headrick, V.E. Hubeny, A. Lawrence, and M. Rangamani, Causality \& holographic entanglement entropy, J. High Energy Phys. 12 (2014) 162.

[38] N. Bao and I. F. Halpern, Holographic inequalities and entanglement of purification, J. High Energy Phys. 03 (2018) 006.

[39] X. Dong, D. Harlow, and A. C. Wall, Reconstruction of Bulk Operators within the Entanglement Wedge in GaugeGravity Duality, Phys. Rev. Lett. 117, 021601 (2016).

[40] K. Umemoto and Y. Zhou, Entanglement of purification for multipartite states and its holographic dual, J. High Energy Phys. 10 (2018) 152.

[41] K. Tamaoka, Entanglement Wedge Cross Section from the Dual Density Matrix, Phys. Rev. Lett. 122, 141601 (2019).

[42] N. Bao and I. F. Halpern, Conditional and multipartite entanglements of purification and holography, Phys. Rev. D 99, 046010 (2019).

[43] R.-Q. Yang, C.-Y. Zhang, and W.-M. Li, Holographic entanglement of purification for thermofield double states and thermal quench, J. High Energy Phys. 01 (2019) 114.

[44] Y. Nomura, P. Rath, and N. Salzetta, Pulling the boundary into the bulk, Phys. Rev. D 98, 026010 (2018).

[45] R. Espíndola, A. Guijosa, and J. F. Pedraza, Entanglement wedge reconstruction and entanglement of purification, Eur. Phys. J. C 78, 646 (2018).

[46] P. Liu, Y. Ling, C. Niu, and J.-P. Wu, Entanglement of purification in holographic systems, J. High Energy Phys. 09 (2019) 071.

[47] J. Harper and M. Headrick, Bit threads and holographic entanglement of purification, J. High Energy Phys. 08 (2019) 101.

[48] K. Babaei Velni, M. R. Mohammadi Mozaffar, and M. H. Vahidinia, Some aspects of entanglement wedge crosssection, J. High Energy Phys. 05 (2019) 200.

[49] B. Amrahi, M. Ali-Akbari, and M. Asadi, Holographic entanglement of purification near a critical point, Eur. Phys. J. C 80, 1152 (2020).

[50] P. Jain and S. Mahapatra, Mixed state entanglement measures as probe for confinement, Phys. Rev. D 102, 126022 (2020).

[51] N. Bao and N. Cheng, Multipartite Reflected Entropy, J. High Energy Phys. 10 (2019) 102.

[52] J. Chu, R. Qi, and Y. Zhou, Generalizations of reflected entropy and the holographic dual, J. High Energy Phys. 03 (2020) 151.

[53] S. Dutta and T. Faulkner, A canonical purification for the entanglement wedge cross-section, J. High Energy Phys. 03 (2021) 178.

[54] Y. Kusuki, J. Kudler-Flam, and S. Ryu, Derivation of Holographic Negativity in $\mathrm{AdS}_{3} / \mathrm{CFT}_{2}$, Phys. Rev. Lett. 123, 131603 (2019).

[55] H.-S. Jeong, K.-Y. Kim, and M. Nishida, Reflected entropy and entanglement wedge cross section with the first order correction, J. High Energy Phys. 12 (2019) 170.

[56] P. Bueno and H. Casini, Reflected entropy, symmetries and free fermions, J. High Energy Phys. 05 (2020) 103.
[57] P. Bueno and H. Casini, Reflected entropy for free scalars, J. High Energy Phys. 11 (2020) 148.

[58] Y. Zou, K. Siva, T. Soejima, R. S. K. Mong, and M. P. Zaletel, Universal Tripartite Entanglement in OneDimensional Many-Body Systems, Phys. Rev. Lett. 126, 120501 (2021).

[59] T. Li, J. Chu, and Y. Zhou, Reflected entropy for an evaporating black hole, J. High Energy Phys. 11 (2020) 155.

[60] J. Kudler-Flam, Y. Kusuki, and S. Ryu, Correlation measures and the entanglement wedge cross-section after quantum quenches in two-dimensional conformal field theories, J. High Energy Phys. 04 (2020) 074.

[61] P. Di Francesco, P. Mathieu, and D. Senechal, Conformal Field Theory, Graduate Texts in Contemporary Physics (Springer-Verlag, New York, 1997).

[62] P. Calabrese, J. Cardy, and E. Tonni, Entanglement entropy of two disjoint intervals in conformal field theory II, J. Stat. Mech. (2011) P01021.

[63] C. Agón and T. Faulkner, Quantum corrections to holographic mutual information, J. High Energy Phys. 08 (2016) 118.

[64] This serves as a lower bound for EOP and RE. However, for RE we even have the stronger bound $S_{R} \geq I_{A B}$ as shown in (2.17) of [53].

[65] S. Bagchi and A. K. Pati, Monogamy, polygamy, and other properties of entanglement of purification, Phys. Rev. A 91, 042323 (2015).

[66] See the Supplemental Material at http://link.aps.org/ supplemental/10.1103/PhysRevLett.127.141604 for a detailed analysis of the methods used for computing MI, EOP and RE between two small, distant subsystems of the fermionic and spin Ising CFT, which includes Refs. [67-69].

[67] B. McCoy, Ising model: Exact results, Scholarpedia 5, 10313 (2010).

[68] H. Casini, C. D. Fosco, and M. Huerta, Entanglement and alpha entropies for a massive Dirac field in two dimensions, J. Stat. Mech. (2005) P07007.

[69] B. Ibinson, N. Linden, and A. Winter, Robustness of quantum Markov Chains, Commun. Math. Phys. 277, 289 (2007).

[70] F. Iglnd and I. Peschel, On reduced density matrices for disjoint subsystems, Europhys. Lett. 89, 40001 (2010).

[71] M. Fagotti and P. Calabrese, Entanglement entropy of two disjoint blocks in XY chains, J. Stat. Mech. (2010) P04016.

[72] A. Coser, E. Tonni, and P. Calabrese, Partial transpose of two disjoint blocks in XY spin chains, J. Stat. Mech. (2015) P08005.

[73] A. Coser, E. Tonni, and P. Calabrese, Spin structures and entanglement of two disjoint intervals in conformal field theories, J. Stat. Mech. (2016) 053109.

[74] B. Windt, A. Jahn, J. Eisert, and L. Hackl, Local optimization on pure Gaussian state manifolds, SciPost Phys. 10, 066 (2021).

[75] L. Hackl, T. Guaita, T. Shi, J. Haegeman, E. Demler, and I. Cirac, Geometry of variational methods: dynamics of closed quantum systems, SciPost Phys. 9, 048 (2020).

[76] J. Hauschild, E. Leviatan, J. H. Bardarson, E. Altman, M.P. Zaletel, and F. Pollmann, Finding purifications with minimal entanglement, Phys. Rev. B 98, 235163 (2018). 